\title{
Relation between QT interval and heart rate New design of physiologically adaptive cardiac pacemaker
}

\author{
A F RICKARDS, J NORMAN \\ From The National Heart Hospital, London
}

SUMMARY The relation between QT interval and heart rate has been studied in a group of patients undergoing physiological exercise, in a group undergoing atrial pacing without exercise, and in a group with complete heart block undergoing exercise at a fixed ventricular rate controlled by cardiac pacing. The expected shortening in QT interval during physiological exercise is only in part the result of the intrinsic effect of increased rate, since patients undergoing atrial pacing to comparable rates show only a small decrease in measured QT interval and patients exercising at fixed rates in heart block exhibit a decreasing QT interval related to the independent atrial rate. QT interval changes appear mainly to be governed by factors extrinsic to heart rate.

The physiological control of QT interval has been used to construct a cardiac pacemaker which senses the interval between the delivered stimulus and the evoked $T$ wave, and uses the stimulusevoked $T$ wave interval to set the subsequent pacemaker escape interval. Thus physiological control of cardiac pacing rate, independent of atrial activity, using conventional unipolar lead systems is possible.

The decrease in QT interval which occurs with exercise-induced changes in heart rate has long been recognised and has led to the use of formulae which correct the measured QT to a basic heart rate. The non-linear relation first described by Bazett $^{1}$ has been widely applied to derive this rate corrected QT interval (QTc) though Bazett himself pointed out the wide variation in individual subjects and only a limited amount of post-exercise data was included in his study. We have previously noted that during diagnostic atrial pacing the observed QT interval appeared to shorten less than during exercise at comparable heart rates. We have therefore re-examined the relation between heart rate and QT interval to determine those factors responsible for the change in ventricular repolarisation time.

\section{Patients and methods}

EXERCISE IN SINUS RHYTHM

A group of 25 patients undergoing a treadmill exercise test was studied to re-evaluate the relation between QT interval and heart rate. All subjects were being investigated for ischaemic heart disease and were exercised according to the Bruce protocol.
Twelve lead electrocardiograph recordings were made at a paper speed of $100 \mathrm{~mm} / \mathrm{s}$ using a Cambridge automatic three-channel recorder complying with AHA standards. Recordings were made at rest, at $90 \mathrm{~s}$ intervals during exercise, immediately after exercise, and at $90 \mathrm{~s}$ intervals after exercise until the resting heart rate was reached. Measurements of rate and QT interval were made from the direct recordings. Data were excluded if a change in the QRS-T morphology occurred either because of artefact, ischaemic changes, conduction defects, or ectopic beats. Data were analysed for the group as a whole and subdivided into those patients taking beta-blocking drugs (15) and those on no cardioactive therapy (10). Further analysis of the rate-QT relation with increasing rate (exercise) and with decreasing rate (recovery) was also made.

ATRIAL PACING

Similar data were obtained from 15 patients undergoing an atrial pacing stress test during cardiac catheterisation for the evaluation of chest pain. Simultaneous 12 lead electrocardiograms were recorded on a Cambridge photographic recorder at the resting rate and during atrial pacing from 100 beats per minute (bpm) in increments of 
$10 \mathrm{bpm}$ until either the maximum rate of $180 \mathrm{bpm}$ was achieved or the test terminated because of chest pain or atrioventricular block. A detailed description of the atrial pacing procedure has been previously reported by ourselves. ${ }^{2}$

\section{EXERCISE AT FIXED HEART RATE}

Nine patients in complete heart block with VVI (ventricular inhibited) pacemakers set at 70 bpm were exercised according to the Bruce protocol. The relation between atrial rate and pacing stimulusQT interval was measured during exercise and recovery using the methods described above, except that by definition the paced QRS complex was wide and showed a left bundle-branch block pattern caused by right ventricular endocardial pacing.

\section{Results}

Fig 1 shows the results obtained during exercise for the patients in sinus rhythm. The data for the group as a whole indicate that the rate-QT interval relation can be approximated by a linear regression equation such that QT interval is predicted by $\mathrm{QT}=522-1.87 \times \mathrm{RATE}$. Thus a rate change of $100 \mathrm{bpm}$ would be accompanied by a change in QT interval of approximately $187 \mathrm{~ms}$. Subdivision of patients on and off beta-blocking agents indicates that though the resting and attained rates are necessarily lower for those patients on betablockers there is no change in the rate-QT relation.

The QT interval does not exhibit hysteresis in relation to rate change, as there is no difference in the rate-QT relation when rate is increasing during exercise or decreasing after exercise.

The data plotted in Fig. 2 were obtained from the patients undergoing atrial pacing to increase heart rate. Though the QT interval decreases with increasing rate, the slope of the rate-QT relation is

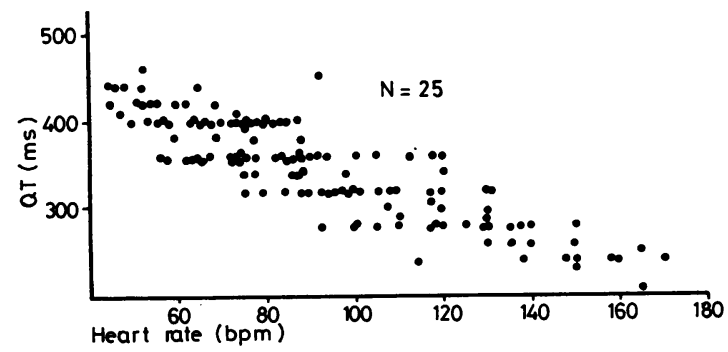

Fig. 1 Data obtained from 25 patients undergoing treadmill exercise. Heart rate is plotted against $Q T$ interval.

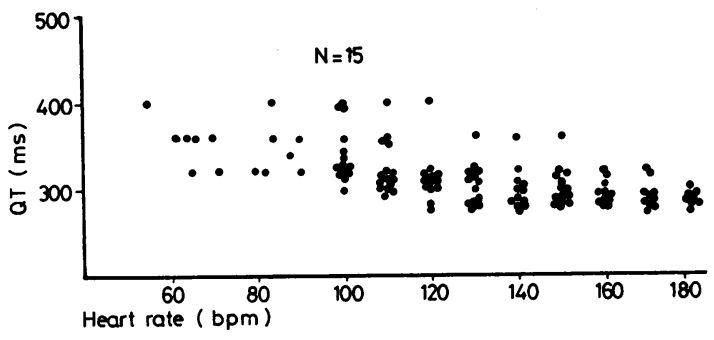

Fig. 2 Data obtained from 15 patients undergoing an atrial pacing stress test at rest under catheter laboratory conditions. Heart rate is plotted against $Q T$ interval.

much flatter (QT $=399-0.66 \times$ RATE). Thus an increase of $100 \mathrm{bpm}$ caused by atrial pacing would produce a QT decrease of approximately $66 \mathrm{~ms}$.

In Fig. 3 the data from the patients in heart block exercised at a fixed ventricular rate controlled by an implanted cardiac pacemaker are plotted. The atrial rate was measured from the electrocardiogram and used as an indicator of the sympathetic and humoral stimulation occurring because of exercise.

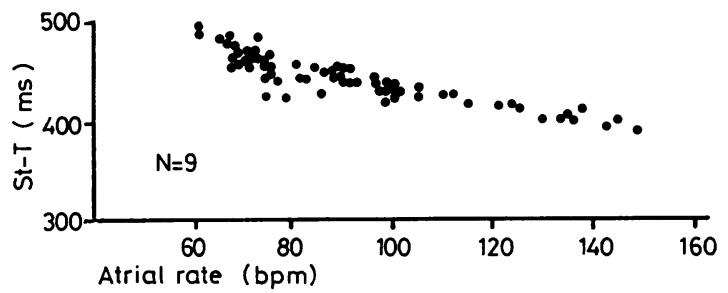

Fig. 3 Data obtained from nine patients in complete heart block exercised with a fixed ventricular rate of 70 bpm. Pacing stimulus- $T$ interval is plotted against the independent atrial rate.

Though the ventricular rate remains unchanged throughout the exercise there is a pronounced decrease in stimulus- $T$ interval with increasing atrial rate $(\mathrm{QT}=530-0.95 \times \mathrm{RATE})$ so that for a $100 \mathrm{bpm}$ atrial rate increase, a decrease in QT interval of $95 \mathrm{~ms}$ would be predicted.

\section{Discussion}

The dichotomy between the QT interval changes caused by physiological exercise and those caused 
by pacing-induced changes in heart rate has been previously described by ourselves. ${ }^{3}$

We have suggested that the sensitivity of the sinus node in the sick sinus syndrome might be determined by examining the relation between sinus rate and QT interval on exercise.

A recent publication from Milne et al. ${ }^{4}$ studying the changes in QT interval induced by propranolol at fixed atrial pacing rates confirms that the QT interval is not only dependent on rate but is also dependent on the change in catecholamine sensitivity of the heart induced by propranolol.

Observations on isolated mammalian cardiac muscle ${ }^{5}$ confirm that catecholamines enhance the rate of relaxation independent of rate, but additionally a purely rate-dependent abbreviation of the action potential has also been demonstrated. ${ }^{6}$ ? Both of these effects are responsible for the abbreviation of systole necessary in the mammalian heart at high rates to maintain adequate diastolic filling.

The results from the atrial pacing study here described indicate that the intact heart does show some sensitivity of repolarisation time to rate, but of a smaller magnitude than during physiological exercise.

From the data obtained when exercising patients in heart block it is apparent that stimulus- $T$ interval is shortening independent of heart rate and therefore presumably as a result of the effects of circulating catecholamines, as there is effectively no direct communication between the autonomic nervous system and the ventricular myocardium. The maintenance of the normal relation between QT interval and rate during exercise under the influence of propranolol implies that the effect of propranolol in blocking the sinus response to circulating catecholamines is accompanied by a similar blocking of the effect of circulating catecholamines on repolarisation time of the ventricular myocardium.
There is little doubt therefore that the QT interval changes observed during exercise are strongly influenced by the effect of circulating catecholamines on the ventricular myocardium and less influenced by the intrinsic alteration in heart rate.

This effect is analogous to the atrial pacinginduced increase in atrioventricular nodal conduction time which is not seen with physiological exercise.

A decrease in QT interval is still seen during atrial pacing and one might argue that this has occurred as a result of the release of endogenous catecholamines resulting from patient anxiety during the procedure. Our previous observations ${ }^{3}$ on the sinus rate after cessation of pacing, however, indicate that this is unlikely, and one must conclude that increasing rate does have an effect on repolarisation time.

These observations naturally led us to consider the use of the QT interval in the design of a physiologically adaptive cardiac pacemaker where the stimulus-evoked $T$ interval is used to assess the "expected" heart rate (as a result of the effect of circulating catecholamines on repolarisation time) and thus set the subsequent pacing interval. Major technical difficulties exist in sensing the ventricular evoked response after a pacing stimulus when using the same electrode for pacing and sensing, and we are unaware of any publications which adequately describe the evoked endocardial signal.

\section{SENSING THE EVOKED RESPONSE}

After delivery of a pacing stimulus above stimulation threshold an electrical response is evoked in the ventricular myocardium which is seen on the surface as a ventricular depolarisation. The evoked response has been assumed to be similar to spontaneous ventricular depolarisations which can easily be sensed by pacing electrodes and whose morphology
Fig. 4 Tracing obtained when a pacing stimulus ( 5.4 volts : $0.5 \mathrm{~ms}: 1 \mathrm{Kohm}$ load) is discharged into a saline bath. A post-stimulus (St) potential decaying over a period of approximately $300 \mathrm{~ms}$ is seen.

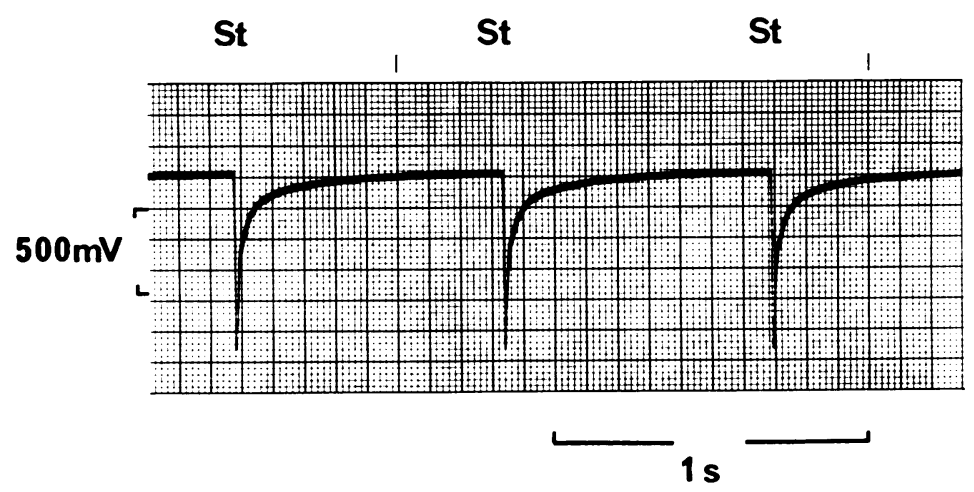


has been well documented and has formed the basis for the design of sensing circuits in non-competitive ventricular pacemakers.

A typical pacing pulse delivers in excess of 5 volts for a time of $0.5 \mathrm{~ms}$ into a load of $500 \mathrm{ohms}$. This stimulus produces polarisation effects at the interfaces between the electrodes and tissue such that after the termination of the pacing pulse itself a decaying voltage exists between cathode and anode of opposite polarity to the pacing stimulus and of a magnitude and time course depending on the energy delivered, the materials employed as cathode and anode, and the surface area of contact between the electrodes and tissue.

Furthermore, most pacemaker output circuits are capacitor coupled in such a way that the capacitor discharges across the electrodes after charging during the pacing pulse.

The summation of these effects is shown in Fig. 4 where a conventional pacemaker and electrode lead system was discharged into a saline bath. The signal measured directly from the electrode lead shows a large potential opposite in polarity to the pacing stimulus with a magnitude of hundreds of millivolts and decaying over a period of more than $300 \mathrm{~ms}$.

It is not surprising therefore that it has proved technically difficult to isolate an evoked response of the order of a few millivolts in magnitude from the post-stimulus period. Conventional pulse generators use absolute refractory periods of the order of 300 to $350 \mathrm{~ms}$ to avoid the polarisation and capacitor discharge effects created at the electrode/heart interface before enabling spontaneous beat sensing.

Preston and Bowers ${ }^{8}$ in 1973 reported the design of a threshold tracking system which sensed the evoked response within $50 \mathrm{~ms}$ of the pacing stimulus and delivered a backup pacing pulse of higher energy if the initial pulse failed to capture the heart. No recordings of the evoked response were demonstrated and subsequent development of this concept was limited by the reliability with which the evoked response could be sensed. Mugica et al. ${ }^{\circ}$ in 1973 used an independent intracardiac electrode to sense the evoked response when pacing and came to the rather surprising conclusion that evoked responses of the order of 500 to 800 millivolts were present 25 to $90 \mathrm{~ms}$ after the pacing pulse.

In 1978 we first developed a pacing input/output circuit which was capable of allowing direct recordings of the cardiac evoked response from a conventional electrode lead system. The system has been refined in collaboration with a commercial organisation ${ }^{\star}$ and depends on a modified pulse waveform and automatic postpulse compensation to

$\star$ Vitatron Medical BV, Dieren, The Netherlands.

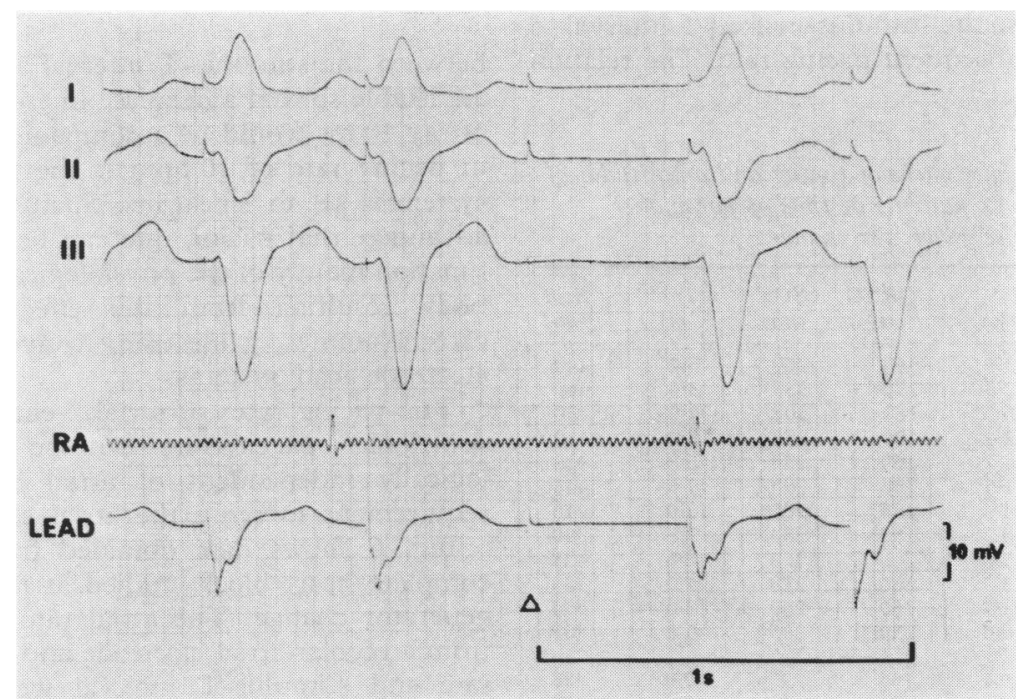

Fig. $5 A$ recording from a patient in complete heart block paced at $70 \mathrm{bpm} . I, I I, I I I=s c a l a r$ leads. $R A$, bipolar atrial electrogram showing independent atrial activity. LEAD, recording from a chronically implanted unipolar lead (Vitatron Helifix) using a stainless steel indifferent electrode in the supramammary pacemaker site. Current, $6.5 \mathrm{~mA}$ at $0.5 \mathrm{~ms}$. Note the negative evoked QRS complex followed by a positive $T$ wave. The arrow indicates the point at which the current was reduced to below stimulation threshold and shows the absence of an evoked response. 
eliminate polarisation and discharge effects. This system is capable of sensing the evoked response within $5 \mathrm{~ms}$ of the pacing pulse delivered across a conventional unipolar lead system and has a truly DC response, so that the evoked response is seen unaffected by any form of filtering. The output circuit has been incorporated into an external ventricular demand pulse generator.

Fig. 5 shows a recording made of the evoked response using this system. The evoked response is seen as a negative QRS complex which reaches a maximum amplitude approximately $45 \mathrm{~ms}$ after the pacing pulse and is followed by a clearly defined positive $T$ wave. Both QRS and $T$ wave duration tend to be shorter than those observed on the surface electrocardiogram but are of higher amplitude than spontaneous complexes sensed by the pacing lead. The Table details observations on the QRS and $T$ durations and amplitudes made in a group of 12 patients studied at pacemaker implantation or pulse generator change.

Both QRS and T wave polarity depend on the sensing dipole. Thus, a positive $T$ wave is sensed by a unipolar endocardial electrode, but the $T$ wave becomes negative if a bipolar endocardial or a unipolar epicardial lead system is used.

\section{ALTERATION OF PACING RATE AS FUNCTION OF STIMULUS-T INTERVAL}

The pacing system has been further refined to incorporate measurement of the peak of the evoked $T$ wave and to use the stimulus-evoked $T$ interval to determine the subsequent pacing rate. The relation

Table Measurements made of timing and amplitudes of evoked response in 12 patients at time of pacemaker implantation or pulse generator change

\begin{tabular}{|c|c|c|c|c|c|c|}
\hline $\begin{array}{l}\text { Case } \\
\text { no. }\end{array}$ & $\begin{array}{l}S t-Q \hat{R} S \\
(m s)\end{array}$ & $\begin{array}{l}\hat{Q R} S \\
(m V)\end{array}$ & $\begin{array}{l}\text { QRSD } \\
\text { (ms) }\end{array}$ & $\begin{array}{l}S t-\hat{T} \\
(m s)\end{array}$ & $\begin{array}{l}\hat{T} \\
(m V)\end{array}$ & $\begin{array}{l}S t-T \\
\text { (ms) }\end{array}$ \\
\hline $\begin{array}{r}1 \\
2 \\
3 \\
4 \\
5 \\
6 \\
7 \\
8 \\
9 \\
10 \\
11 \\
12\end{array}$ & $\begin{array}{l}38 \\
60 \\
44 \\
\\
42 \\
45 \\
45 \\
44 \\
55 \\
40 \\
36 \\
40\end{array}$ & $\begin{array}{l}-23 \\
-12 \cdot 5 \\
-16 \\
-22 \cdot 4 \\
-14 \cdot 7 \\
-11 \cdot 5 \\
-14 \\
-18 \cdot 9 \\
-15 \\
-13 \\
-21 \cdot 7 \\
-10 \cdot 0\end{array}$ & $\begin{array}{l}160 \\
170 \\
180 \\
180 \\
160 \\
150 \\
155 \\
150 \\
200 \\
180 \\
160 \\
150\end{array}$ & $\begin{array}{l}360 \\
385 \\
308 \\
250 \\
280 \\
290 \\
280 \\
280 \\
320 \\
270 \\
250 \\
285\end{array}$ & $\begin{array}{r}3 \cdot 2 \\
3.0 \\
7 \cdot 0 \\
3.2 \\
8 \cdot 8 \\
7 \cdot 5 \\
12.0 \\
7 \cdot 8 \\
8 \cdot 8 \\
5.0 \\
13.0 \\
5.0\end{array}$ & $\begin{array}{l}440 \\
460 \\
430 \\
410 \\
410 \\
445 \\
410 \\
390 \\
425 \\
380 \\
350 \\
405\end{array}$ \\
\hline $\begin{array}{l}8 \\
S D\end{array}$ & $\begin{array}{r}44 \cdot 5 \\
7 \cdot 2\end{array}$ & $\begin{array}{l}-16.1 \\
-4.4\end{array}$ & $\begin{array}{r}166 \\
16\end{array}$ & $\begin{array}{r}297 \\
41\end{array}$ & $\begin{array}{l}7 \cdot 0 \\
3 \cdot 3\end{array}$ & $\begin{array}{r}413 \\
30\end{array}$ \\
\hline
\end{tabular}

St- $\mathrm{QRS}=$ time from pacing stimulus to peak $\mathrm{QRS}$.

$\mathrm{QR} S=$ peak $\mathrm{QRS}$ amplitude. $\mathrm{QRSD}=\mathrm{QRS}$ duration.

$S t-T=$ time from stimulus to peak of $T$ wave. $T=$ peak $T$ wave amplitude. St- $T=$ time from stimulus to end of $T$ wave. $\mathrm{X}=$ mean values. $\mathrm{SD}=$ standard deviation of mean.
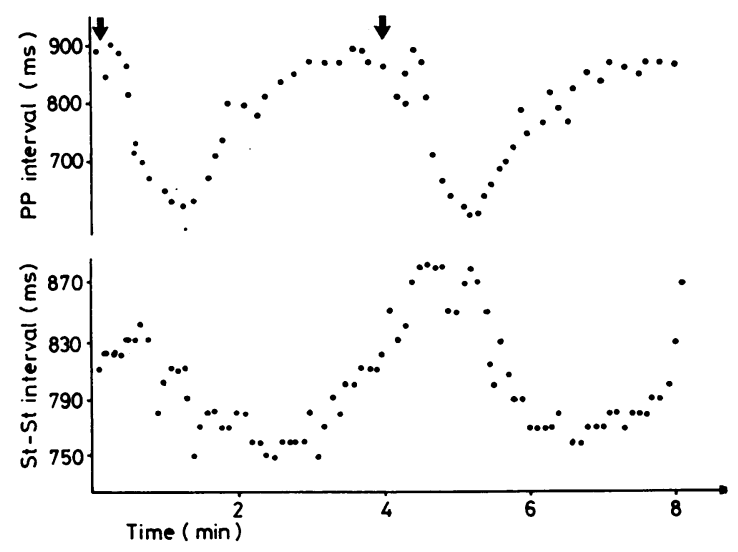

Fig. 6 A plot of PP interval and stimulusstimulus interval against time in a patient with complete heart block. Each point represents a 6 s rate average. The arrows indicate the intravenous injection of $2 \mu \mathrm{g}$ isoprenaline. Pacing rate-stimulus $T$ sensitivity was set at 0.4 beats/ms. Note the increase in atrial rate (decrease in PP interval) which reaches a maximum approximately $90 \mathrm{~s}$ after isoprenaline. The pacing system responds to the decreasing stimulus-evoked $T$ interval and increases rate (decreasing $S t-S t$ interval) to a maximum approximately one minute after the peak atrial response. Both atrial and pacemaker responses are complete at five minutes and the isoprenaline challenge then repeated.

between the stimulus- $T$ interval and pacing rate is adjustable so that a decrease in stimulus- $T$ interval of say $10 \mathrm{~ms}$ would be accompanied by an increase in pacing rate of $10 \mathrm{bpm}$ if the sensitivity of the system is set to 1 beat $/ \mathrm{ms}$. Such a system has the advantage that pacing rate can be determined as a function mainly of the physiological demands of the body resulting from the effect of circulating catecholamines, while using conventional unipolar electrode lead systems.

The pacing rates of atrial, ventricular, or atrioventricular pacemakers can be altered physiologically independent of atrial activity and the requirement to sense the atrial electrogram.

Fig. 6 shows data obtained from a patient in complete heart block studied at the time of pulse generator change. The atrial rate was determined from a bipolar atrial electrode and changes in atrial rate and stimulus- $T$ interval were produced by intravenous injections of $2 \mu \mathrm{g}$ isoprenaline.

Alterations in the pacing rate can be seen to follow stimulation with isoprenaline. The effect of isoprenaline on the stimulus- $T$ interval and subsequent increase in pacing rate is delayed by 
about one minute in relation to the effect on sinus rate, but an increase in pacing rate as a result of isoprenaline with recovery over a period of five minutes is clearly shown and is reproducible.

\section{Conclusions}

Data are presented which show that the decrease in QT interval seen during exercise occurs not primarily as a result of an increase in heart rate but mainly results from the effects of circulating catecholamines which accompany exercise. This sensitivity of the repolarisation of the ventricular myocardium has been used to design a pacing system which offers the advantage of physiological control of pacing rate, independent of atrial activity, using conventional endocardial electrode lead systems.

The authors wish to express their thanks to Vitatron Medical BV and especially to Mr A C M Renirie and $\mathrm{Mr}$ F H M Wittkampf for help in the development of this concept.

\section{References}

1 Bazett HC. An analysis of the time-relations of electrocardiograms. Heart 1920; 7: 353-70.
2 Rickards AF, Seabra-Gomes R. Observations on the effect of angina on the left ventricle with special reference to diastolic behaviour. Eur $\mathcal{F}$ Cardiol 1978; 7, suppl: 213-38.

3 Rickards AF, Akhras F, Baron DW. Effects of heart rate on QT interval (abstract). In: Meere C, ed. Proceedings of the VI world symposium on cardiac pacing. Montreal: Laplante \& Langevin, 1979; 2: 7.

4 Milne JR, Camm AJ, Ward DE, Spurrell RAJ. Effect of intravenous propranolol on QT interval. A new method of assessment. Br Heart $\mathcal{F} 1980$; 43: 1-6.

5 Morad M, Rolett E. Relaxing effects of catecholamines on mammalian heart. $f$ Physiol 1972; 224: 537-58.

6 Hoffman BF, Cranefield P. Electrophysiology of the heart. New York: McGraw-Hill, 1960.

7 Boyett MR, Jewell BR. A study of the factors responsible for rate-dependent shortening of the action potential in mammalian ventricular muscle. f Physiol 1978; 285: 359-80.

8 Preston TA, Bowers DL. Report of a continuous threshold tracing system. In: Thalen HJTh, ed. Cardiac pacing. Assen: Van Gorcum, 1973: 295-9.

9 Mugica J, Lazarus B, Buffet J, Catte M. Pacemaker with automatic adaptation to the pacing threshold. In: Thalen HJTh, ed. Cardiac pacing. Assen: Van Gorcum, 1973: 150-5.

Requests for reprints to Dr Anthony Rickards, The National Heart Hospital, Westmoreland Street, London W1M 8BA. 